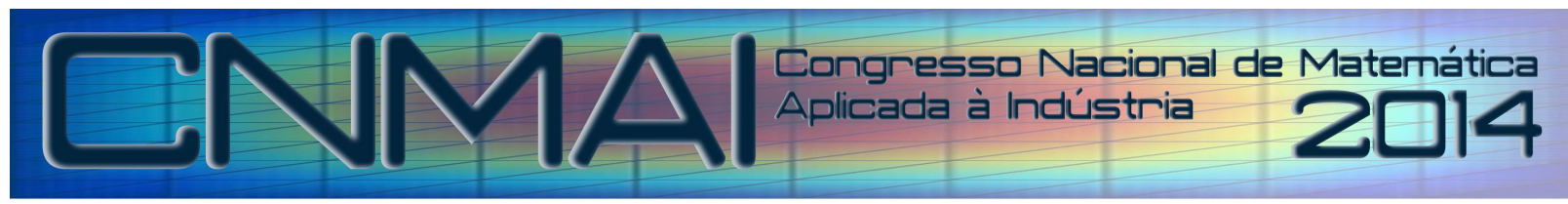

18 a 21 de novembro de 2014, Caldas Novas - Goiás

\title{
DETERMINAÇÃO DOS PARÂMETROS DE INTERAÇÃO DO SISTEMA TERNÁRIO ACETATO DE ETILA/ÁGUA/AÇÚCARR USANDO O ALGORITMO DE EVOLUÇÃO DIFERENCIAL
}

\author{
Flavio Caldeira Silva, flaviocaldeirasilva@ hotmail.com $^{1}$ \\ Fran Sérgio Lobato, fslobato@feq.ufu.br ${ }^{1}$ \\ Moilton Ribeiro Franco Jr, moilton@ufu.br ${ }^{1}$
}

${ }^{1}$ Faculdade de Engenharia Mecânica, Universidade Federal de Uberlândia

Av. João Naves de Ávila, 2121, Campus Santa Mônica, P.O. Box 593, 38400-902, Uberlândia-MG, Brasil.

\begin{abstract}
Resumo: $O$ conhecimento do comportamento termodinâmico de misturas é uma informação relevante durante o projeto de equipamentos empregados em processos de separação. Tradicionalmente para a representação do equilíbrio de fases de misturas envolvidas nestes processos são empregados modelos de coeficiente de atividade. Esses por sua vez possuem parâmetros de interação intermoleculares que precisam ser determinados a partir de dados experimentais de equilíbrio. A determinação destes parâmetros caracteriza um problema inverso, isto é, um problema de estimação de parâmetros. Neste cenário, o presente trabalho tem por objetivo a determinação dos parâmetros de interação do sistema ternário acetato de etila/água/açúcar usando o Algoritmo de Evolução Diferencial com subpopulações. Para essa finalidade utilizou-se dados experimentais de equilíbrio para sistemas ternários e modelos tradicionais de coeficiente de atividade em temperaturas distintas. Foram estimados parâmetros dos modelos de Wilson, NRTL e UNIQUAC. Os resultados obtidos indicam que metodologia proposta configura-se como uma interessante alternativa para a resolução do problema inverso proposto.
\end{abstract}

Palavras-chave: Problema Inverso, Parâmetros de Iteração, Algoritmo de Evolução Diferencial, Sub-Populações.

\section{INTRODUÇÃO}

Nos últimos anos, a análise inversa tem-se caracterizado como uma ferramenta de grande aplicabilidade em vários campos da ciência e da engenharia. Esta tem como fundamentação básica a formulação e a resolução de um problema de otimização através do uso de dados experimentais. No contexto dos projetos de sistemas de engenharia, esta tem-se configurado como uma boa alternativa para a determinação de parâmetros de equações constitutivas utilizados nas equações de balanços de massa, energia e quantidade de movimento, de modo que os modelos possam ser utilizados para simular o comportamento do sistema de interesse em diferentes condições de operação.

Na engenharia química, o conhecimento do comportamento termodinâmico de misturas constitui uma informação de grande relevância para o projeto de equipamentos de separação, tais como colunas de destilação. Tradicionalmente, a representação do equilíbrio de fases de misturas de componentes envolvidos nestes processos é caracterizado por modelos que fazem uso de coeficientes de atividade (Walas, 1995; Reid et al., 1988; Smith et al., 2007). Estes por sua vez possuem parâmetros de interação intermoleculares que precisam ser determinados a partir de dados experimentais de equilíbrio. A determinação destes parâmetros caracteriza um problema inverso. Conceitualmente, define-se problema inverso como sendo aquele que, de posse de informações da saída do processo, pode-se formular um problema de otimização de modo a resgatar informações sobre a entrada e/ou sobre o modelo que gerou a respectiva saída considerada.

Tradicionalmente, a resolução dos problemas inversos tem sido tratada via aplicação de métodos fundamentados no uso de derivadas (Métodos Determinísticos). Estes apresentam como principais vantagens (Vanderplaats, 1999): $i$ ) fácil concepção conceitual e fácil implementação, ii) alta taxa de convergência. Por outro lado, como desvantagens pode-se citar (Vanderplaats, 1999; Lobato, 2008): i) dificuldade no tratamento de problemas com descontinuidades, com a presença de variáveis mistas (inteiras, contínuas, discretas, binárias) e com restriçõos de caminho, ii) não são capazes de escapar de ótimos locais, e iii) podem ser sensíveis a escolha da estimativa inicial, entre outros.

Nas últimas décadas, para superar as dificuldades encontradas na aplicação de Métodos Determinísticos (MD), a literatura especializada tem dedicado esforços para o desenvolvimento e aprimoramento dos Métodos NãoDeterminísticos (MND), isto é, técnicas de otimização que não fazem uso de informações sobre o gradiente da função objetivo e de suas restrições. De forma geral, estes apresentam as seguintes vantagens (Lobato, 2008): $i$ ) fácil concepção conceitual e fácil implementação, ii) são capazes de escapar de ótimos locais, sendo desta forma, caracterizados como métodos de busca global, iii) são capazes de lidar com descontinuidades e com problemas com 
restrições de caminho, $i v$ ) podem ser facilmente expandidos para tratar problemas com variáveis mistas, e $v$ ) trabalham com uma população de candidatos a solução do problema de otimização, isto é, não investem todo o esforço computacional em um único ponto, como acontece nos MD. Por outro lado, como desvantagens pode-se citar (Lobato, 2008): $i$ ) elevado número de avaliações da função objetivo em comparação com os $\mathrm{MD}$, e $i i$ ) a solução ótima é função da escolha da semente inicial considerada no gerador de números aleatórios considerado, o que requer que o mesmo seja executado várias vezes com diferentes sementes inicias para a obtenção de valores médios.

Dentre estes métodos, destaca-se o algoritmo de Evolução Diferencial (ED) proposto por Storn e Price (1995). Basicamente, este algoritmo se diferencia de outras abordagens não-determinísticas pela forma como os candidatos a solução do problema de otimização são gerados, isto é, através de operações vetoriais simples. Apesar da simplicidade desta técnica, na literatura especializada podem ser encontradas várias aplicações do algoritmo de ED, dentre as quais pode-se citar: a estimação de parâmetros cinéticos no processo de fermentação batelada (Wang et al., 2001), o controle ótimo multi-objetivo de um fermentador batelada alimentada (Lobato et al., 2007), o projeto de sistemas de engenharia (Lobato e Steffen Jr, 2007), o problema inverso de transferência de calor por radiação (2009ab), a estimação da difusividade térmica na secagem de frutas (Mariani et al., 2008), a estimação de parâmetros térmicos em um secador rotativo (Arruda et al., 2008), a minimização da energia livre de Gibbs em sistemas reais (2009b), dentre outras (Storn et al., 2005).

Diante do que foi apresentado, o presente trabalho tem por objetivo a determinação dos parâmetros de interação do sistema ternário acetato de etila/água/açúcar usando o algoritmo de ED com sub-populações $\left(\mathrm{ED}_{\mathrm{sp}}\right)$. Para essa finalidade considera-se dados experimentais de equilíbrio para sistemas ternários e modelos tradicionais de coeficiente de atividade em temperaturas distintas (Modelos de Wilson, NRTL - Non-Random Two-Liquid Model, e UNIversal QUAsiChemical - UNIQUAC) (Walas, 1995; Reid et al., 1988; Smith et al., 2007). Este trabalho esta estruturado como segue. A seção 2 apresenta a descrição do processo estudado. As seções 3 e 4 apresentam a descrição dos algoritmos $\mathrm{ED}$ e $\left(\mathrm{ED}_{\mathrm{sp}}\right)$, bem como apresentar a formulação do problema inverso considerado neste trabalho. Já nas seções 5 e 6 são apresentados a metodologia e os resultados obtidos com o algoritmo de ED em comparação com o Método de Levenberg-Marquardt (MLM). Finalmente, as conclusões são apresentadas na última seção.

\section{DESCRIÇÃO DO PROCEDIMENTO EXPERIMENTAL}

As medidas experimentais de solubilidade foram realizadas utilizando uma técnica com base na experiência de grupo de pesquisa que desenvolve estudos a respeito de equilíbrios sólido-líquido e líquido-líquido (Oliveira et al., 2007-2008; Malagoni e Franco, 2007; Pires e Franco, 2008; Oliveira, 2009). Foram usadas células de vidro encamisadas interligadas por mangueiras de latex e conectados a um banho termostático da marca Nova Ética - Modelo $521-2$, o qual mantêm a temperatura do sistema constante (precisão $\pm 0,1^{\circ} \mathrm{C}$ ). As células de equilíbrio possuíam volume de cerca de $40 \mathrm{~cm}^{3}$ e foram carregadas com a quantidade apropriada de compostos, açúcar e água, para realizar a desejada concentração 10,0-30,0 g.. $\mathrm{L}^{-1}$. Cada solução de diferente composição foi preparada com a pesagem da quantidade desejada de cada composto em uma balança analítica de precisão $\pm 0,0001 \mathrm{~g}$. $\mathrm{O}$ acetato de etila foi adicionado à solução de maneira que um ligeiro excesso fosse garantido em relação à solubilidade do esperado, com uma solução de açúcar especificado, glicose ou sacarose. A Figura 1 apresenta o aparato experimental empregado para a determinação das condições de equilíbrio.

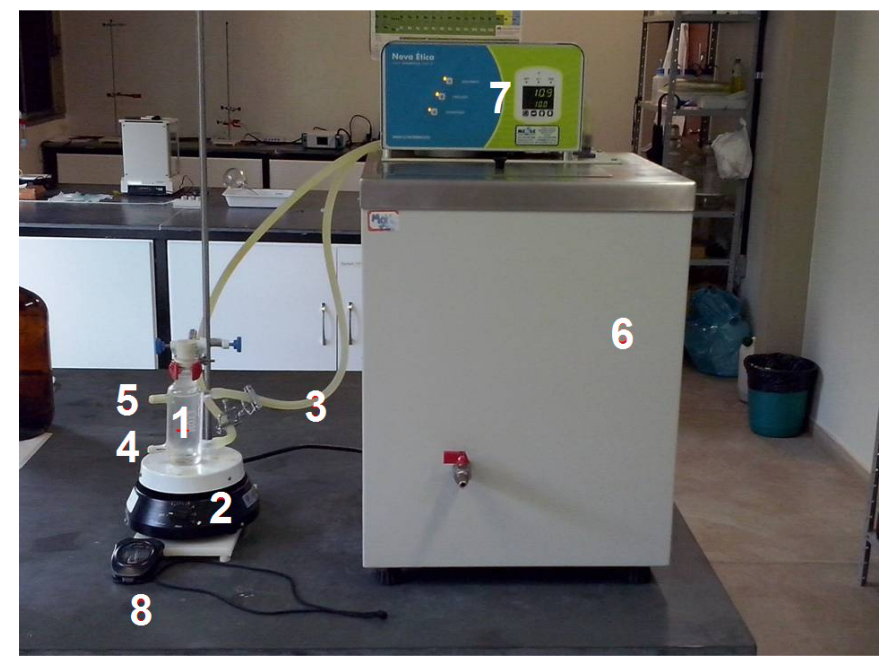

Figura 1. Aparato experimental: (1) Célula de equilíbrio; (2) agitador magnético; (3) mangueiras de látex; (4) e (5) septos de borracha; (6) banho termostatizado; (7) Indicador e controle de temperatura e (8) cronômetro digital. 
Após cerca de 2-3 h de agitação e 24 horas de decantação, a fase aquosa foi amostrada e analisada, em triplicata, por cromatografia gasosa, empregando detector de ionização de chama. Curva de calibração apropriada foi obtida para determinação final do valor de solubilidade do orgânico no equilíbrio.

\section{ALGORITMO DE EVOLUÇÃO DIFERENCIAL}

O algoritmo ED é uma versão melhorada dos atuais algoritmos genéticos para a resolução de problemas de otimização (Babu et al., 2005). A principal ideia por trás desta técnica heurística é o esquema proposto para atualização do vetor de variáveis de projeto que constitui uma população de candidatos em potencial. Basicamente, a diferença ponderada entre dois indivíduos da população é adicionada a um terceiro indivíduo. O indivíduo gerado através deste esquema é avaliado pela função objetivo e pode inclusive substituir indivíduos mal sucedidos nas gerações seguintes. Desta forma, nenhuma distribuição de probabilidade em separado deve ser usada, o que torna este esquema completamente auto-ajustável.

Os parâmetros de controle no algoritmo de ED são: $N$ o tamanho da população, $C R$ a probabilidade de cruzamento, e $D$ a taxa de perturbação. O algoritmo canônico é apresentado a seguir (Storn et al., 2005).

\section{Evolução Diferencial}

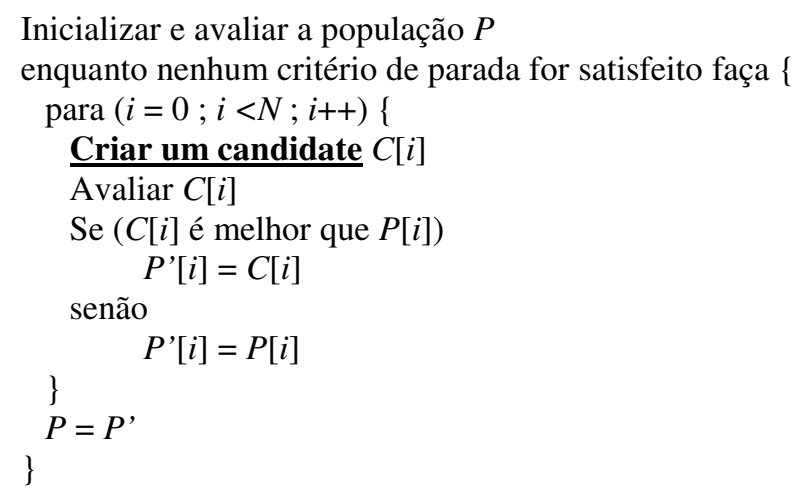

onde $N$ é o tamanho da população, $P$ é a população da geração (iteração) corrente, e $P$ ' é a população da próxima geração. A rotina Criar um candidato $C[i]$ é descrita a seguir.

\section{Criar um candidato $\mathrm{C}[i]$}

Randomicamente selecione os pais $P\left[i_{1}\right], P\left[i_{2}\right]$, e $P\left[i_{3}\right]$ onde $i, i_{1}, i_{2}$, e $i_{3}$ são diferentes.

Crie inicialmente o candidato $C_{1}[i]=P\left[i_{1}\right]+D \times\left(P\left[i_{2}\right]-P\left[i_{3}\right]\right)$.

Crie o candidate final $C[i]$ pelo cruzamento dos genes de $P[i]$ e $C_{1}[i]$ como mostrado a seguir:

para $(j=0 ; j<N ; j++)\{$

se $(r<C R)$

$C[i][j]=C_{1}[i][j]$

senão

\}

$C[i][j]=\mathrm{P}_{1}[i][j]$

$C[i]$ é a solução candidate com índice de população $i, C[i][j]$ é a $j$-th entrada no vetor de solução de $C[i], r$ é um número randômico entre 0 e 1 .

\subsection{Algoritmo de Evolução Diferencial com Sub-Populações $\left(\mathbf{E D}_{\mathrm{sp}}\right)$}

Como em qualquer técnica de otimização, a solução final obtida é função da estimativa inicial considerada. Neste contexto, a depender da escolha da estimativa inicial pode-se ter convergência (lenta ou rápida) ou divergência. Assim, toda e qualquer melhoria para aumentar a chance de convergência em tais métodos é sempre bem vinda. Em se tratando de MND, inúmeras estratégias para essa finalidade já foram propostas. Intuitivamente, sabe-se que uma população de candidatos é atualizada de acordo com estratégia que cada método é fundamentado. Neste caso, a depender da escolha da semente inicial considerada no gerador de números aleatórios, a população pode estagnar em torno de um ponto que não é a solução ótima global. Para superar tal dificuldade, o presente trabalho propõe o uso de sub-populações. Basicamente, a estratégia denominada de $\mathrm{ED}_{\mathrm{sp}}$ consiste em definir um número $n$ de sub-populações, onde cada uma delas evoluirá independentemente das outras, através da aplicação individual do algoritmo de ED. Assim, depois de um certo número de gerações, as informações contidas em cada uma destas sub-populações são trocadas, isto é, as populações são "embaralhadas". Tal procedimento, apesar de simples, inseri em cada população uma certa 
aleatoriedade, de modo a estagnação de cada sub-população seja minimizada, aumentando desta forma, a chance da obtenção da solução ótima global.

\section{FORMULAÇÃO DO PROBLEMA INVERSO}

Matematicamente, o problema inverso considerado neste trabalho consiste na determinação dos parâmetros de interação de modo a minimizar o funcional $F$, conforme definido a seguir:

$$
F=\sum_{i=1}^{n_{\mathrm{exp}}} f_{i}^{2}
$$

onde $f_{i}$ é a equação algébrica definida pelo modelo termodinâmico considerado (Wilson, NRTL ou UNIQUAC) e $n_{\exp }$ é o número de pontos experimentais considerados. Se o número de graus de liberdade for igual a zero, isto é, o número de equações for igual ao número de pontos experimentais, o problema consiste de um sistema de equações não lineares. Por outro lado, se o número de pontos experimentais for superior ao número de parâmetros que devem ser estimados, o problema final a ser resolvido é um de otimização.

\section{METODOLOGIA}

Como são conhecidos apenas dois pontos experimentais nas temperaturas de $5^{\circ} \mathrm{C}$ e $25^{\circ} \mathrm{C}$, para a determinação dos parâmetros de interação dos sistemas ternários, deve-se primeiramente encontrar os parâmetros de interação dos sistemas binários correspondentes. Neste caso são dois sistemas binários em equilíbrio sólido-líquido (ESL), glicose e água e sacarose e água, e um sistema binário em equilíbrio líquido-líquido (ELL), acetato de etila e água. Para a determinação dos parâmetros do sistema ELL, utilizou-se dados experimentais de equilíbrio e modelos de coeficiente de atividade a diluição infinita. Já para os sistemas ESL, utilizaram-se os dados experimentais e as equações dos modelos para cálculo dos coeficientes de atividade, conforme descrição a seguir.

\subsection{Modelo de Wilson}

Na equação desenvolvida por Wilson (Walas, 1995; Reid et al., 1988; Smith et al., 2007). a temperatura aparece como variável explícita, permitindo admitir que os parâmetros sejam dela independentes (Renon e Prausnitz, 1968). Neste modelo a energia livre de Gibbs de excesso tem como referência uma solução ideal segundo a lei de Raoult e nos extremos de composição (componentes puros) $G_{\text {sol }}^{E}$ tende a zero. O modelo se aplica a vários tipos de misturas, e é particularmente útil para soluções de compostos com tendência à associação, como pontes de hidrogênio e polaridade. A equação de Wilson apresenta também como vantagem o fato de ser facilmente estendida para soluções multicomponentes. O termo $\Lambda_{i j}$ está diretamente ligado a uma grandeza que relaciona a fração molar local à fração molar bulk, originada da teoria da composição local, estabelecida para determinados casos, como quando há forças radicalmente diferentes de atração, onde a interação da mistura pode ser fortemente associada a composição da mistura, $V_{i}$ e $V_{j}$, volumes molares das moléculas, $R$ é a constante dos gases e $T$, temperatura absoluta.

Da termodinâmica, tem-se que o Modelo de Wilson para um sistema ternário é dado por:

$$
\begin{gathered}
\ln \left(Y_{1}\right)=1-\ln \left(x_{1}+x_{2} \Lambda_{12}+x_{3} \Lambda_{13}\right)-\left(\frac{x_{1}}{x_{1}+x_{2} \Lambda_{12}+x_{3} \Lambda_{13}}+\frac{x_{2} \Lambda_{21}}{x_{1} \Lambda_{21}+x_{2}+x_{3} \Lambda_{23}}+\frac{x_{3} \Lambda_{31}}{x_{1} \Lambda_{31}+x_{2} \Lambda_{23}+x_{3}}\right) \\
\Lambda_{i j}=\frac{V_{i}}{V_{j}} \exp \left(-\frac{a_{i j}}{R T}\right)
\end{gathered}
$$

em que os componentes 1, 2 e 3 dizem respeito ao acetato e etila, água e açúcar, respectivamente, $x$ é fração molar do componente $i, \Lambda_{i j}$ é o parâmetro de interação binária do componente $i$ em relação ao componente $j, V_{i}$ é o volume molar do componente $i, R$ é a constante geral dos gases e $T$ é a temperatura. A equação a seguir é utilizada para a determinação dos volumes molares dos componentes puros (Reid et al., 1988):

$$
V_{i}=0,285 V_{c i}^{1,048}
$$

onde $V_{c i}$ é o volume crítico da substância $i\left(V_{c 1}=286,00 \mathrm{~cm}^{3} \mathrm{~mol}^{-1} \mathrm{e} V_{c 2}=55,95 \mathrm{~cm}^{3} \mathrm{~mol}^{-1}\right)$.

\subsection{Modelo NRTL}

O modelo NRTL, desenvolvido por Renon e Prausnitz (1968), também está fundamentado no conceito de composição local, a diferença é que este pode ser aplicado a sistemas de miscibilidade parcial, o que não é possível para a equação de Wilson. O modelo NRTL apresenta três parâmetros ajustáveis, onde dois deles $\left(g_{i j}\right)$ com significado 
similar aos $l_{i j}$ da equação de Wilson, ou seja, parâmetros de energia característicos das interações $i$-j, e um parâmetro $a_{i j}$ relacionado com a aleatoriedade da mistura de forma que quando $a_{i j}$ é zero, a mistura é completamente randômica, e a equação se reduz à equação de Margules de dois parâmetros. O modelo NRTL fornece uma boa representação dos dados experimentais para diversos sistemas, porém é necessário dados de boa qualidade e precisão devido à existência dos três parâmetros a serem ajustados.

Matematicamente, o modelo NRTL um sistema ternário é dado por:

$$
\begin{gathered}
\ln \left(Y_{1}\right)=\frac{x_{2} \tau_{21} G_{21}+x_{3} \tau_{31} G_{31}}{x_{1}+x_{2} G_{21}+x_{3} G_{31}}+\frac{x_{1}}{x_{1}+x_{2} G_{21}+x_{3} G_{31}}\left(-\frac{x_{2} \tau_{21} G_{21}+x_{3} \tau_{31} G_{31}}{x_{1}+x_{2} G_{21}+x_{3} G_{31}}\right)+ \\
+\frac{x_{2} G_{12}}{x_{1} G_{12}+x_{2}+x_{3} G_{32}}\left(\tau_{12}-\frac{x_{1} \tau_{12} G_{12}+x_{3} \tau_{32} G_{32}}{x_{1} G_{12}+x_{2}+x_{3} G_{32}}\right)+\frac{x_{3} G_{13}}{x_{1} G_{13}+x_{2} G_{23}+x_{3}}\left(\tau_{13}-\frac{x_{1} \tau_{13} G_{13}+x_{2} \tau_{23} G_{23}}{x_{1} G_{13}+x_{2} G_{23}+x_{3}}\right) \\
G_{i j}=\exp \left(-\alpha \tau_{i j}\right) \\
\tau_{i j}=\frac{g_{i j}}{R T}
\end{gathered}
$$

em que $\alpha$ é o parâmetro de não aleatoriedade da mistura, obtido experimentalmente e tem como valor usual 0,3 , e $G_{i j}$ e $\tau_{i j}$ são os parâmetros dados pelas Eq. (6) e (7).

\subsection{Modelo UNIQUAC}

O modelo UNIQUAC de Abrams e Prausnitz (1975) foi desenvolvido com base nos trabalhos de Wilson, com três refinamentos principais. Primeiramente, a dependência da temperatura da função é modificada, dependendo das áreas de superfície ao invés de depender dos volumes, baseado na hipótese de que as energias de interações que determinam as composições locais são dependentes das áreas de superfície relativas das moléculas. Segundo, os diferentes tamanhos e formas das moléculas são considerados implicitamente na equação da energia, e qualitativamente, o número de moléculas que podem entrar em contato com uma molécula central aumenta com o aumento do tamanho da molécula. A terceira diferença da equação de Wilson está na avaliação da constante de integração da equação da energia livre de Helmholtz. Essa contribuição (independente da temperatura) é atribuída a entropia de mistura de cadeias rígidas, já que grandes moléculas não são necessariamente grandes esferas, mas as vezes cadeias longas. A forma atualmente aplicada no método UNIQUAC é a de Guggenheim. Notando que a razão entre a área de superfície e o volume de uma esfera difere da razão com o volume de uma cadeia, Guggenheim fornece uma correção simples, porém genérica, dando uma indicação do grau de ramificação e da não-esfericidade das moléculas.

A equação UNIQUAC para $G^{E}$ (Energia de Gibbs excedente) consiste em duas partes: uma parte combinatorial, que descreve as contribuições entrópicas dos componentes, e uma parte residual, que expressa as forças intermoleculares que são responsáveis pela entalpia de mistura. A parte combinatorial depende apenas da composição e do tamanho e forma das moléculas, necessita apenas de dados do componente puro; no entanto, a parte residual depende das forças intermoleculares, de onde aparecem os dois parâmetros ajustáveis. UNIQUAC é aplicável a uma ampla variedade de misturas líquidas não-eletrolíticas, contendo componentes polares e não polares, incluindo sistemas de miscibilidade parcial.

Matematicamente, o modelo UNIQUAC para um sistema ternário é dado por:

$$
\begin{gathered}
\tau_{12}=\exp \left(k_{8}-\exp \left(k_{7}-\tau_{12}\right)\right) \\
\ln \left(Y_{1}\right)=\ln \left(\frac{\Phi_{1}}{x_{1}}\right)+5 q_{1} \ln \left(\frac{\Phi_{1}}{x_{1}}\right)+L_{1}-\frac{\Phi_{1}}{x_{1}}\left(x_{1} L_{1}+x_{2} L_{2}+x_{3} L_{3}\right)+ \\
+q\left(1-\ln \left(\theta_{1}+\theta_{2} \tau_{12}+\theta_{3} \tau_{13}\right)\right)-\frac{\theta_{1}}{\theta_{1}+\theta_{2} \tau_{21}+\theta_{3} \tau_{31}}-\frac{\theta_{2} \tau_{12}}{\theta_{1} \tau_{12}+\theta_{2}+\theta_{3} \tau_{32}}-\frac{\theta_{3} \tau_{13}}{\theta_{1} \tau_{13}+\theta_{2} \tau_{23}+\theta_{3}} \\
\Phi_{i}=\frac{x_{i} r_{i}}{x_{i} r_{i}+x_{j} r_{j}} \\
\theta_{i}=\frac{x_{i} q_{i}}{x_{i} q_{i}+x_{j} q_{j}} \\
q_{i}=\sum_{k} v_{k}^{i} Q_{k} \\
r_{i}=\sum_{k} v_{k}^{i} R_{a k}
\end{gathered}
$$




$$
L_{i}=\frac{z}{2}\left(r_{i}-q_{i}\right)-r_{i}-1
$$

Para a resolução do problema inverso definido pela Eq. (1) serão empregadas as seguintes abordagens: $i$ ) Método de Levenberg-Marquardt - MLM (para resolver o sistema de equações não lineares resultantes da aplicação dos modelos termodinâmicos), ii) Algoritmo de Evolução Diferencial - ED (canônico) e iii) Algoritmo de Evolução Diferencial com sub-populações - $\mathrm{ED}_{\mathrm{sp}}$.

\section{RESULTADOS E DISCUSSÃO}

As Tabelas 1 e 2 apresentam algumas informações, que foram obtidas da literatura, fundamentais para a determinação dos parâmetros de interação, dos modelos de coeficientes de atividade para o sistema ternário acetato de etila/água/açúcar. Resultados de concentração de açúcar em água, em duas temperaturas de equilíbrio, podem ser obtidas da Tabela 1.

Tabela 1. Dados experimentais de solubilidade de açúcares em água em diferentes temperaturas.

\begin{tabular}{c|cc|cl|c}
\cline { 2 - 5 } & \multicolumn{4}{|c|}{ Solubilidade } & Fonte \\
\hline Sacarose & $\mathrm{XS}=0,04258$ & $\mathrm{~T}=25^{\circ} \mathrm{C}$ & $\mathrm{XS}=0,11$ & $\mathrm{~T}=40^{\circ} \mathrm{C}$ & Peres e Macedo (1997) \\
\hline Glicose & $\mathrm{XG}=0,149$ & $\mathrm{~T}=30^{\circ} \mathrm{C}$ & $\mathrm{XG}=0,175$ & $\mathrm{~T}=40^{\circ} \mathrm{C}$ & Gaida et al. (2006) \\
\hline
\end{tabular}

$\mathrm{XS} \rightarrow$ fração molar da sacarose no binário sacarose-água; $\mathrm{XG} \rightarrow$ fração molar da glicose no binário glicose-água; XF $\rightarrow$ fração molar da frutose no binário frutose-água.

Para aplicação da equação de equilíbrio sólido-líquido, para os binários açúcar-água, é necessário o conhecimento das grandezas ilustradas na Tabela 2.

Tabela 2. Dados termodinâmicos para cálculos da solubilidade de Glicose e Sacarose em água.

\begin{tabular}{c|c|c}
\hline & D-Glicose & D-Sacarose \\
\hline & $416,15^{\mathrm{a}}$ & $463,15^{\mathrm{a}}$ \\
Ponto de Fusão (K) & $423,15^{\mathrm{b}}$ & $458,15^{\mathrm{b}}$ \\
& $419,15^{\mathrm{c}}$ & $459,15^{\mathrm{c}}$ \\
\hline \multirow{2}{*}{ Entalpia de fusão $\left(\mathrm{J} \mathrm{mol}^{-1}\right)$} & $32248^{\mathrm{a}}$ & $40391^{\mathrm{a}}$ \\
& $32432^{\mathrm{b}}$ & $41076^{\mathrm{b}}$ \\
\hline$\Delta \mathrm{A}\left(\mathrm{J} \mathrm{mol}^{-1} \mathrm{~K}^{-1}\right)$ & $31432^{\mathrm{c}}$ & $46187^{\mathrm{c}}$ \\
\hline$\Delta \mathrm{B}\left(\mathrm{J} \mathrm{mol}^{-1} \mathrm{~K}^{-1}\right)$ & $139,5766^{\mathrm{d}}$ & $316,1153^{\mathrm{d}}$ \\
${ }^{\mathrm{a}}$ & $0^{\mathrm{d}}$ & $-1,1547^{\mathrm{d}}$ \\
\hline${ }^{\mathrm{c}}$ Roos (1993) apud Peres e Macedo (1996), ${ }^{\mathrm{b}}$ Raemy e Schweizer (1983) apud Peres e Macedo (1996),
\end{tabular}

Para a resolução do problema inverso formulado foram considerados os seguintes parâmetros nos algoritmos ED e $\mathrm{ED}_{\mathrm{sp}}$ : 50 indivíduos (somente no $\mathrm{ED}$ ) distribuídos em duas sub-populações (somente no $\mathrm{ED}_{\mathrm{sp}}$ ); 250 gerações; probabilidade de cruzamento e taxa de perturbação iguais a 0,8 e vetor de sementes iniciais $\left[\begin{array}{lllll}0 & 1 & 2 & \ldots & 9\end{array}\right]$. Neste caso, considerando os parâmetros definidos, são requeridas, em cada execução dos referidos algoritmos, $50+50 \times 250$ avaliações da função objetivo. Para confrontar os resultados obtidos com a metodologia proposta será empregado o MLM com diferentes condições iniciais. Para essa metodologia, o número de avaliações da função é dependente do critério de parada considerado, que neste caso foi o valor numérico do sistema de equações for menor que a tolerância de $10^{-8}$.

Como primeira aplicação, será considerado a estimação dos parâmetros de interação binário $\left(a_{12}\right.$ e $\left.a_{21}\right)$ do sistema acetato de etila (1) e água (2) para o Modelo de Wilson através do MLM e dos algoritmos ED e ED sp. Para este caso, o modelo matemático a ser resolvido via MLM e pelos dos algoritmos $\mathrm{ED}$ e $\mathrm{ED}_{\mathrm{sp}}$ são dados respectivamente pelas Eq. (15) e Eq. (16):

$$
\begin{gathered}
\left\{\begin{array}{l}
\ln \left(Y_{1}^{\infty}\right)=-\ln \left(\frac{V_{1}}{V_{2}} \exp \left(-\frac{a_{12}}{R T}\right)\right)+\left(1-\frac{V_{2}}{V_{1}} \exp \left(-\frac{a_{21}}{R T}\right)\right) \\
\ln \left(Y_{2}^{\infty}\right)=-\ln \left(\frac{V_{2}}{V_{1}} \exp \left(-\frac{a_{21}}{R T}\right)\right)+\left(1-\frac{V_{1}}{V_{2}} \exp \left(-\frac{a_{12}}{R T}\right)\right)
\end{array}\right. \\
\min _{a_{12}, a_{21}}\left(\begin{array}{l}
\left(-\ln \left(\frac{V_{1}}{V_{2}} \exp \left(-\frac{a_{12}}{R T}\right)\right)+\left(1-\frac{V_{2}}{V_{1}} \exp \left(-\frac{a_{21}}{R T}\right)\right)-\ln \left(Y_{1}^{\infty}\right)\right)^{2}+ \\
\left.\left(-\ln \left(\frac{V_{2}}{V_{1}} \exp \left(-\frac{a_{21}}{R T}\right)\right)+\left(1-\frac{V_{1}}{V_{2}} \exp \left(-\frac{a_{12}}{R T}\right)\right)-\ln \left(Y_{2}^{\infty}\right)\right)^{2}\right)
\end{array}\right.
\end{gathered}
$$


onde a $Y_{1}^{\infty}$ e $Y_{2}^{\infty}$ são os coeficientes de atividade (66,8 e 10,7) (Reid et al., 1988), $T$ é a temperatura (50+273,15 K), $R$ é a constante de gases $(1,987 \mathrm{cal} / \mathrm{mol} \mathrm{K}), V_{c 1}$ e $V_{c 2}$ são os volumes críticos dos componentes 1 e 2 , respectivamente (286 e $55,9 \mathrm{~cm}^{3} / \mathrm{mol}$ ). Assim, os valores de $V_{1}$ e $V_{2}$ são computados pela equação (4).

A Tab. (3) apresenta os resultados obtidos com as três abordagens citadas.

Tabela 3. Parâmetros de interação binário do sistema acetato de etila (1) e água (2) considerando o Modelo de Wilson via MLM e os algoritmos ED e ED $\mathbf{E D}_{\mathrm{sp}}$.

\begin{tabular}{|c|c|c|}
\hline Metodologia & Estimativa Inicial & $A_{12}(\mathrm{cal} / \mathrm{mol}) / a_{21}(\mathrm{cal} / \mathrm{mol}) / F$ (Eq. (1)) \\
\hline \multirow{5}{*}{ MLM } & {$\left[\begin{array}{ll}0 & 0]\end{array}\right.$} & $1115,34 / 1998,75 / 10^{-17}$ \\
\hline & {$\left[\begin{array}{ll}1 & 1\end{array}\right]$} & $1115,34 / 1998,76 / 10^{-16}$ \\
\hline & {$\left[\begin{array}{lll}100 & 100\end{array}\right]$} & $1115,34 / 1998,75 / 10^{-17}$ \\
\hline & {$[91,854,38]$} & $\mathrm{real} / \mathrm{complexo} / 10^{-8}$ \\
\hline & {$[68,5715,32]$} & $\mathrm{real} / \mathrm{complexo} / 10^{-8}$ \\
\hline \multirow{11}{*}{ ED } & Semente & $A_{12}(\mathrm{cal} / \mathrm{mol}) / a_{21}(\mathrm{cal} / \mathrm{mol}) / F($ Eq. $(1))$ \\
\hline & 0 & $10729,41 / 1978,33 / 2,25 \times 10^{-4}$ \\
\hline & 1 & $10729,41 / 1978,33 / 2,25 \times 10^{-4}$ \\
\hline & 2 & $1115,42 / 1998,78 / 2,01 \times 10^{-8}$ \\
\hline & 3 & $957,33 / 12926,40 / 8,73 \times 10^{-3}$ \\
\hline & 4 & $1115,42 / 1998,78 / 2,01 \times 10^{-8}$ \\
\hline & 5 & $10729,41 / 12926,40 / 8,96 \times 10^{-3}$ \\
\hline & 6 & $10729,41 / 12926,40 / 8,96 \times 10^{-3}$ \\
\hline & 7 & $10729,41 / 1978,33 / 2,25 \times 10^{-4}$ \\
\hline & 8 & $957,32 / 12926,40 / 8,74 \times 10^{-3}$ \\
\hline & 9 & $957,31 / 12926,39 / 8,73 \times 10^{-3}$ \\
\hline \multirow{11}{*}{$\mathrm{ED}_{\mathrm{sp}}$} & Semente & $A_{12}(\mathrm{cal} / \mathrm{mol}) / a_{21}(\mathrm{cal} / \mathrm{mol}) / F($ Eq. (1)) \\
\hline & 0 & $1115,34 / 1998,76 / 10^{-19}$ \\
\hline & 1 & $1115,34 / 1998,76 / 10^{-19}$ \\
\hline & 2 & $1115,34 / 1998,76 / 10^{-19}$ \\
\hline & 3 & $1115,34 / 1998,76 / 10^{-19}$ \\
\hline & 4 & $1115,34 / 1998,76 / 10^{-19}$ \\
\hline & 5 & $1115,34 / 1998,76 / 10^{-19}$ \\
\hline & 6 & $1115,34 / 1998,76 / 10^{-19}$ \\
\hline & 7 & $1115,34 / 1998,75 / 10^{-19}$ \\
\hline & 8 & $1115,34 / 1998,75 / 10^{-19}$ \\
\hline & 9 & $1115,34 / 1998,75 / 10^{-19}$ \\
\hline
\end{tabular}

Na Tabela 3 é possível observar a sensibilidade do MLM e do algoritmo ED na resolução do problema inverso proposto, sendo a qualidade da solução altamente influenciada pela escolha da condição inicial/semente considerada. Todavia, essa flutuação em relação a melhor solução encontrada pelo MLM não é observada nos resultados obtidos pelo algoritmo $\mathrm{ED}_{\mathrm{sp}}$. Isto se deve ao mecanismo que foi incorporado ao algoritmo canônico, permitindo que as informações contidas em cada uma das duas sub-populações consideradas sejam trocadas de modo a aumentar a de diversidade dos candidatos. Assim, apesar de simples, tal mecanismo aumenta as chances das sub-populações não se tornarem homogêneas ou ficarem estagnadas em torno de um ótimo local, promovendo desta forma, uma busca mais eficiente.

A seguir são apresentados os resultados obtidos pelo MLM (estimativa inicial igual ao vetor nulo) e pelo algoritmo de $\mathrm{ED}_{\mathrm{sp}}$ (considerando o vetor de sementes iniciais definido anteriormente) para todos os modelos termodinâmicos, bem como para todos os sub-sistemas considerados.

A partir dos resultados apresentados na Tab. 4 é possível concluir que ambas as estratégias utilizadas conseguiram encontrar resultados satisfatórios em termos do valor da função objetivo (Eq. (1)), quando comparados com aqueles obtidos pelo Método de Levenberg-Marquardt. 
Tabela 4. Parâmetros de interação dos sistemas considerando todos os modelos termodinâmicos via aplicação do MLM e do algoritmo $\mathbf{E D}_{\text {sp. }}$

\begin{tabular}{|c|c|c|c|}
\hline Modelo & Metodologia & Sistema & $a_{12} / a_{21} / a_{23} / a_{32} / a_{13} / a_{31}(\mathrm{cal} / \mathrm{mol})$ \\
\hline \multirow{4}{*}{ Wilson } & MLM & \multirow{2}{*}{$\begin{array}{l}\text { Acetato de etila (1)/ } \\
\text { Água (2)/Glicose (3) }\end{array}$} & $1115,34 / 1998,75 /-1078,59 / 384,75 /-3613,21 /-5332,59$ \\
\hline & $\mathrm{ED}_{\mathrm{sp}}$ & & $1115,34 / 1998,75 /-1078,58 / 384,74 /-3613,19 /-5332,62$ \\
\hline & MLM & Acetato de etila (1)/ & $1115,34 / 1998,75 / 37,66 /-43,73 /-2296,90 /-4630,44$ \\
\hline & $\mathrm{ED}_{\mathrm{sp}}$ & Água (2)/Sacarose (3) & $1115,34 / 1998,75 / 37,65 /-43,73 /-2296,89 /-4630,43^{*}$ \\
\hline \multirow{4}{*}{ NRTL } & MLM & \multirow{2}{*}{$\begin{array}{l}\text { Acetato de etila (1)/ } \\
\text { Água (2)/Glicose (3) }\end{array}$} & $734,67 / 2176,70 / 366,68 /-963,85 / 2335,93 / 1911,35$ \\
\hline & $\mathrm{ED}_{\mathrm{sp}}$ & & $734,67 / 2176,70 / 366,67 /-963,84 / 2335,92 / 1911,36^{*}$ \\
\hline & MLM & Acetato de etila (1)/ & $734,67 / 2176,70 / 931,18 /-1154,58 / 2963,60 / 1875,07$ \\
\hline & $\mathrm{ED}_{\mathrm{sp}}$ & Água (2)/Sacarose (3) & $734,67 / 2176,70 / 931,18 /-1154,55 / 2963,60 / 1875,06^{*}$ \\
\hline \multirow{4}{*}{ UNIQUAC } & MLM & \multirow{2}{*}{$\begin{array}{l}\text { Acetato de etila (1)/ } \\
\text { Água (2)/Glicose (3) }\end{array}$} & $-150,43 /-438,35 / 399,23 /-207,34 / 166,83 / 409,43$ \\
\hline & $\mathrm{ED}_{\mathrm{sp}}$ & & $-150,43 /-438,35 / 399,23 /-207,34 / 166,80 / 409,42^{*}$ \\
\hline & MLM & \multirow{2}{*}{$\begin{array}{l}\text { Acetato de etila (1)/ } \\
\text { Água (2)/Sacarose (3) }\end{array}$} & $-150,43 /-438,35 /-136,87 / 140,72 /-298,63 / 75,77$ \\
\hline & $\mathrm{ED}_{\mathrm{sp}}$ & & $-150,43 /-438,35 /-136,88 / 140,70 /-298,60 / 75,78^{*}$ \\
\hline
\end{tabular}

*Valores médios encontrados considerando todas as execuções.

\section{CONCLUSÕES}

Este trabalho teve por objetivo determinar os parâmetros de interação do sistema ternário acetato de etila/água/açúcar (glicose e sacarose) através da formulação e resolução de um problema inverso. Este foi formulado a partir do uso de dados experimentais associados a modelos termodinâmicos para a construção de um sistema de equações não lineares que devem ser resolvidas simultaneamente. Para essa finalidade considerou-se duas abordagens,

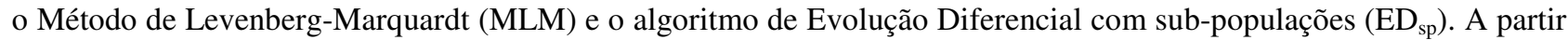
dos resultados obtidos foi possível concluir que os resultados obtidos pelo algoritmo $\mathrm{ED}_{\mathrm{sp}}$ sempre foram superiores aos encontrados pelo MLM e pelo algoritmo ED. Nos casos onde aplicou-se o MLM e o algoritmo ED observou-se que os mesmos são sensíveis a escolha da estimativa inicial, conduzindo desta forma para soluções locais. Já o algoritmo $\mathrm{ED}_{\mathrm{sp}}$, observa-se que, independentemente da escolha da semente inicial, o algoritmo sempre foi capaz de obter a melhor solução. Isto se deve ao procedimento de diversidade de soluções através troca de informações entre as sub-populações consideradas.

Em termos dos modelos termodinâmicos considerados, a escolha do melhor, para o sistema considerado, pode ser realizada a partir da predição dos valores de solubilidade para temperaturas na faixa de $5^{\circ} \mathrm{C}$ a $25^{\circ} \mathrm{C} \operatorname{com}$ os parâmetros determinados neste trabalho.

Como sequência deste trabalho, pretende-se avaliar a qualidade dos modelos termodinâmicos considerados. Além disso, testar outros sistemas multi-componentes e formular um problema inverso com mais pontos obtidos experimentalmente.

\section{AGRADECIMENTOS}

Os autores agradecem a FAPEMIG e ao CNPq pelo suporte financeiro.

\section{REFERÊNCIAS}

Abrams, D.S.; Prausnitz, J.M.,1975. Statistical thermodynamics of liquid mixtures: A new expression for the excess Gibbs energy of partly or completely miscible systems, AIChE Journal, v. 21, 116-128.

Arruda, E. B., Lobato, F. S., Barrozo, M. A. S., Steffen Jr, V., 2008. Estimation of drying parameters in rotary dryers using differential evolution. 6th International Conference on Inverse Problems in Engineering: Theory and Practice - ICIPE, Dourdan-França.

Babu, B. V.; Chakole, P. G.; Mubben, J. H. S., 2005. Multiobjective differential evolution (mode) for optimization of adiabatic styrene reactor. Chemical Engineering Science, v. 60, p. 4822-4837.

Gaida, L.; Dussap, J. B., 2006. Variable hydration of small carboydrates for predicting equilibrium properties in diluted and concentrated solutions. Food Chemistry, V. 96, p. $387-401$.

Lobato, F. S., 2008. Otimização Multi-objetivo para o Projeto de Sistemas de Engenharia. Tese de Doutorado, Universidade Federal de Uberlândia.

Lobato, F. S., Figueira, C. E. Soares, R. R., Steffen Jr., V., 2009b. A comparative study of Gibbs free energy minimization in real systems using heuristic methods. 10th International Symposium on Process Systems Engineering - PSE'09, Salvador - Ba, August, 16-20.

Lobato, F. S., Murata, V. V., Oliveira-Lopes, L. C., Steffen Jr, V., 2007. Solution of multi-objective optimal control problems with Index fluctuation using differential evolution. 6th Brazilian Conference on Dynamics, Control and Their Applications - DINCON, São José do Rio Preto-Brasil. 
Lobato, F. S., Silva Neto, A. J., Steffen Jr., V., 2009a. Adaptive differential evolution based on the concept of population diversity applied to simultaneous estimation of radiation phase function, albedo and optical thickness. 20th International Congress of Mechanical Engineering - COBEM, Gramado-RS.

Lobato, F. S., Steffen Jr, V., 2007. Engineering system design with multi-objective differential evolution, In Proceedings in 19th International Congress of Mechanical Engineering - COBEM.

Malagoni, R. A.; Franco, M. R., Jr. 2007. A Simple Apparatus for Data Solubility Determination. Fluid Phase Equilib. v. 255, p. 93-97.

Mariani, V. C., Lima, A. G. B., Coelho, L. S., 2008. Apparent thermal diffusivity estimation of the banana during drying using inverse method, Journal of Food Engineering, 85, 569-579.

Oliveira, A. C.; Pires, R. F.; Coelho, M. G.; Franco, M. R. Jr., 2007. Solubility of Benzoic Acid in Mixed Solvents. J. Chem. Eng. Data, v. 52, p. 298-300.

Oliveira, M. L. N. 2009. Estudo experimental da solubilidade de alguns ácidos em misturas hidro-alcoólicas. (Tese de doutorado). Faculdade de Engenharia Química, Universidade Federal de Uberlândia, Uberlândia, 322 p.

Oliveira, M. L. N.; Malagoni, R. A.; Fehr, M.; Franco, M. R., Jr. 2008. Obtaining Solubility Data from a Liquid-Liquid Equilibrium Cell. Chem. Eng. Commun., v.195, p.1076-1084.

Peres, A. M.; Macedo; E. A., 1996. Thermodynamic properties of sugars in aqueous: correlation and prediction using UNIQUAC model, Fluid Phase Equilibria, v. 123, p. 71 - 95.

Pires, R. F.; Franco, M. R., Jr. 2008. Solubility of Benzoic Acid in Aqueous Solutions Containing Ethanol or NPropanol. J. Chem. Eng. Data v. 53, p. 2704-2706.

Reid, R. C., Prausnitz, J. M., Poling, B. E. The Properties of Gases and Liquids. International Edition, ed.4, 1988.

Renon, H.; Prausnitz, J.M., 1968. Local Compositions in Thermodynamics Excess Functions for Liquid Mixtures. AIChE Journal, 14, p. 135-144.

Smith, J. M., van Ness, H. C., Abbott, M. M. Introdução à Termodinâmica da Engenharia Química. Sétima Edição, Rio de Janeiro, 2007.

Storn, R, Price, K., Lampinen, J. A., 2005. Differential Evolution - A Practical Approach to Global Optimization. Springer - Natural Computing Series.

Storn, R., Price, K., 1995. Differential evolution: a simple and efficient adaptive scheme for global optimization over continuous spaces. International Computer Science Institute, vol. 12, pp. 1-16.

Vanderplaats, G. N., 1999; Numerical Optimization Techniques for Engineering Design, Vanderplaats Research and Development, Inc., 3rd ed.

Walas, S. M. Phase Equilibria in Chemical Engineering. Department of Chemical and Petroleum Engineering, University of Kansas and The C.W. Nofsinger Company, 1985.

Wang, F. S., Su, T. L., Jang, H. J., 2001. Hybrid differential evolution for problems of kinetic parameter estimation and dynamic optimization of an ethanol fermentation process. Industry Engineering Chemical Research, vol. 40, pp. 2876-2885.

\title{
RESPONSABILIDADE AUTORAL
}

Os autores são os únicos responsáveis pelo conteúdo deste trabalho.

\section{DETERMINATION OF INTERACTION PARAMETERS IN ETHYL ACETATE/WATER/SUGAR TERNARY SYSTEM USING DIFFERENTIAL EVOLUTION}

\begin{abstract}
The thermodynamic behavior of mixtures is information important in engineering systems design used in separation process. Traditionally, for representation of mixtures phase equilibrium involved in these process the activity coefficient are used. In this context, these parameters should be estimated through the formulation and solution of an inverse problem considering experimental data. In this contribution, the Differential Evolution Algorithm with sub-populations is used to determine the interaction parameters of ethyl acetate/water/sugar ternary system. For this purpose, experimental data for this equilibrium system to formulate the inverse problem and Wilson, NRTL and UNIQUAC models are considered. The results are compared with those obtained by the Levenberg-Marquardt Method.
\end{abstract}

Keywords: Inverse Problem, Iterations Parameters, Differential Evolution Algorithm, Sup-Population. 